\title{
Chitosan gel containing polymeric nanocapsules: a new formulation for vaginal drug delivery
}

\author{
This article was published in the following Dove Press journal: \\ International Journal of Nanomedicine \\ 28 June 2014 \\ Number of times this article has been viewed
}

\author{
Luiza A Frank' \\ Giuseppina Sandri² \\ Francesca D'Autilia ${ }^{2}$ \\ Renata $V$ Contri' \\ Maria Cristina Bonferoni ${ }^{2}$ \\ Carla Caramella ${ }^{2}$ \\ Alejandro G Frank ${ }^{3}$ \\ Adriana R Pohlmann' \\ Silvia S Guterres' \\ 'Pharmaceutical Science Graduate \\ Program, Federal University of Rio \\ Grande do Sul, Porto Alegre, RS, \\ Brazil; ${ }^{2}$ Department of Drug Sciences, \\ University of Pavia, Pavia, PV, Italy; \\ ${ }^{3}$ Department of Industrial Engineering, \\ Federal University of Rio Grande do \\ Sul, Porto Alegre, RS, Brazil
}

\begin{abstract}
The vaginal route of administration is an alternative for several treatments for either local or systemic pharmacological effects. However, the permanence of a drug in this route represents a challenge for formulation development that can be overcome by using nanoencapsulation and chitosan gel. Thus, this work aimed to evaluate the performance of chitosan hydrogels containing cationic and anionic acrylic-based nanocapsules (Eudragit ${ }^{\mathbb{R}}$ RS 100 and Eudragit ${ }^{\circledR}$ S 100, respectively) with Nile red as a model of lipophilic substance in the vaginal route of administration, as measured by increases in the residence time and the penetration of these formulations. Several formulations were prepared with increasing chitosan concentrations, and were analyzed in terms of $\mathrm{pH}$ and rheological behavior so that the most suitable formulation could be selected. The enhancement of the adhesion (tensile stress test and washability profile) and penetration (confocal laser scanning microscopy and extraction followed by quantification) properties of the formulations, when applied to porcine vaginal mucosa, were evaluated. The nanocapsule suspensions produced presented adequate properties: size of approximately $200 \mathrm{~nm}$ (polydispersity index of $\leq 0.2$ ); zeta potential around $+10 \mathrm{mV}$ for the cationic formulation and $-10 \mathrm{mV}$ for the anionic formulation; and $\mathrm{pH}$ values of $6.1 \pm 0.1$ (Eudragit RS 100), $5.3 \pm 0.2$ (Eudragit S 100), 6.2 \pm 0.1 (Nile red loaded Eudragit RS 100), and 5.1 \pm 0.1 (Nile red loaded Eudragit S 100). The chitosan formulation presented suitable viscosity for vaginal application and acidic $\mathrm{pH}$ (approximately 4.5). The tensile stress test showed that both formulations containing polymeric nanocapsules presented higher mucoadhesion when compared with the formulation without nanocapsules. In the washability experiment, no significant differences were found between formulations. Confocal microscopy and fluorescence quantification after extraction from the mucosa showed higher penetration of Nile red when it was nanoencapsulated, particularly in cationic nanocapsules. The formulations developed based on chitosan gel vehicle at $2.5 \%$ weight/weight containing polymeric nanocapsules, especially the cationic nanocapsules, demonstrated applicability for the vaginal delivery of hydrophobic substances.
\end{abstract}

Keywords: Eudragit ${ }^{\circledR}$ RS 100, Eudragit ${ }^{\circledR}$ S 100, vaginal route, nanotechnology

\section{Introduction}

Vaginal delivery is an alternative for several pharmacological treatments for either local or systemic effect. ${ }^{1,2}$ However, the local effect is targeted more often as a method of administration of antibacterials, antifungals, antivirals, antiprotozoals, spermicidal agents, and steroids. ${ }^{1,3}$ Using the vaginal mucosa as a drug delivery route has advantages such as facility of drug application, high contact surface area, high blood supply, good permeability to several substances, and avoidance of first pass metabolism and gastrointestinal side effects. ${ }^{2,4,5}$ However, the development of formulations for application via the vaginal route presents some difficulties. One of these difficulties is that the histology and physiology of the vagina vary with age, menstrual cycle, and hormonal changes. The other difficulty is that the vagina secretes endometrial fluids.
Correspondence: Silvia S Guterres Faculdade de Farmácia, Universidade Federal do Rio Grande do Sul, Av lpiranga, 2752/405 CEP 90610-000 Porto Alegre, RS, Brazil

Tel +55 5I 33085500

Fax +55 5I 33085247

Email silvia.guterres@ufrgs.br 
Such difficulties contribute to easy removal of the formulation, which leads to shorter periods between applications and, therefore, to a low compliance with the treatment. ${ }^{2}$

Several authors have demonstrated that the use of nanotechnology can reduce the above-mentioned disadvantages of vaginal drug administration. ${ }^{6-10}$ This is because, when drugs are encapsulated into nanostructures, their release is controlled, they are protected against degradation, and the dose applied can be eventually reduced. ${ }^{11,12}$ Additionally, it must be considered that some drugs administered via the vaginal route can cause side effects, such as erythema, irritation, ulceration, and pain, and such effects could be reduced by using nanotechnology. ${ }^{5,13}$

Nanoparticles have strong tissue-adhesion properties, which is helpful for therapy because it increases the residence time of drugs. However, nanoparticles are frequently obtained in aqueous suspensions, so application at a specific site is difficult. Therefore, incorporating nanoparticles into a viscous system is an alternative that facilitates the correct delivery of the substance. Moreover, the use of a bioadhesive vehicle, such as chitosan, can prolong residence time on the mucosa, leading to the expected action, ${ }^{2,14,15}$ improving treatment acceptability, and increasing penetration and/or absorption. ${ }^{13,16}$

The use of semisolid formulations represents an alternative method to increase the period of contact between the drug and the mucosa. ${ }^{2,3}$ Additionally, the use of bioadhesive vehicles, such as gels, tablets, suppositories, and films, can improve the action of several drugs. ${ }^{5}$ Different polymers have been described in the literature for the delivery of substances on the vaginal mucosa, such as: polyethylene glycol, cellulose-derivatives, and chitosan. ${ }^{2}$ Among these, chitosan is the only polymer that presents positive charge, which leads to a probable interaction with the vaginal mucosa, increasing the residence time of drugs that contribute to increased mucosae penetration. It also presents other interesting properties, such as biocompatibility, biodegradability, mucoadhesion, antimicrobial activity, and film formation, and the ability to heal injured tissues.

Chitosan, a bioadhesive polymer, has received a great deal of attention in the last few years. ${ }^{3,4,17}$ This polymer is obtained through the deacetylation of chitin, one of the most abundant polysaccharides found in nature. ${ }^{3,18,19}$ The mentioned polymer has been widely applied in the development of different pharmaceutical dosage forms, such as hydrogels, ${ }^{3,4}$ films, ${ }^{13}$ microspheres, ${ }^{20}$ and nanoparticles. ${ }^{21}$ Chitosan hydrogels can be produced by covalent interactions between chitosan chains. ${ }^{22}$ Among the therapeutic applications of the chitosan hydrogel, the controlled release of drugs is particularly notable. ${ }^{22}$ Additionally, chitosan hydrogels affect the tight junction between epithelial cells such that these formulations may increase drug penetration. ${ }^{2}$

The use of chitosan vehicles and nanoparticles for vaginal delivery have been reported by some scholars. ${ }^{3,4,23}$ For instance, hydrogels formed by chitosan derivatives have shown higher adhesion to pig vaginal mucosa than a commercial gel. ${ }^{3}$ The citrate salt of chitosan has been proven to be suitable for the preparation of gels incorporating substances with low permeation potential for vaginal administration. ${ }^{4}$ For example, nanoparticles prepared by ionic gelation containing tenofovir, an antiviral agent, were applied to pig vaginal mucosa. The chitosan formulation presented a high potential for the release of antimicrobial agents due to controlled release, bioadhesion, and safety. ${ }^{23}$

The incorporation of nanostructured systems, such as nanocapsules, into chitosan gels represents the unification of different systems with interesting properties, as previously mentioned. The incorporation of Eudragit ${ }^{\circledR}$ RS 100 nanocapsules (Evonik Industries AG, Essen, Germany) in chitosan hydrogels and the controlled-release property of the formulation has already been described. ${ }^{24}$ The formulation has shown high skin adhesion ${ }^{17}$ and no skin irritation in humans. ${ }^{13}$ In addition to cutaneous administration, use of Eudragit RS 100 nanocapsules has been described for ocular $^{25}$ and vaginal ${ }^{26}$ routes of administration.

The aim of this work was to evaluate the performance of chitosan hydrogels containing cationic and anionic acrylicbased nanocapsules (Eudragit RS 100 and Eudragit ${ }^{\circledR}$ S 100 [Evonik Industries AG], respectively), with Nile red (NR) as a model lipophilic substance, for vaginal administration, as measured by increased residence time and penetration of these formulations.

\section{Materials and methods Materials}

Chitosan of medium molecular weight $(1,136 \mathrm{kDa})$ and $92 \%$ deacetylation degree, 90\% lactic acid, NR, polysorbate 80 , sorbitan monostearate 60, and acetone (analytical grade) were purchased from Sigma-Aldrich (St Louis, MO, USA). Capric/caprylic triglycerides (Miglyol 812) and Eudragit (S 100 and RS 100) were gifts from Sasol (Johannesburg, South Africa) and Evonik Industries AG, respectively. Isopentyl acetate was obtained from Carlo Erba Reagents (Milan, Italy).

\section{Production of nanocapsules}

Nanocapsules were produced by the method described by Fessi et a ${ }^{27}$ called interfacial deposition of preformed polymers, using Eudragit RS $100^{24}$ or Eudragit S $100^{28}$ as 
the polymeric shell $(10 \mathrm{mg} / \mathrm{mL})$, capric/caprylic triglycerides as the oily core $(33 \mu \mathrm{g} / \mathrm{mL})$, polysorbate 80 as the stabilizer $(7.6 \mathrm{mg} / \mathrm{mL})$, and NR $(0.0825 \mathrm{mg} / \mathrm{mL}$, previously mixed with the capric/caprylic triglycerides) as a model of lipophilic drug. Sorbitan monostearate $(7.6 \mathrm{mg} / \mathrm{mL})$ was used for the production of the Eudragit S 100 formulation. The nanocapsules produced with different polymers containing NR were named NC-RS-NR (prepared with Eudragit RS 100) and NC-S-NR (prepared with Eudragit S 100). Similarly, nanocapsule suspensions without the fluorescent marker were used as controls and named NC-RS (Eudragit RS 100) and NC-S (Eudragit S 100).

\section{Characterization of nanocapsules}

The nanocapsules were characterized in terms of $\mathrm{pH}$, size, and zeta potential immediately after production. The $\mathrm{pH}$ analysis was performed by direct measurement using potentiometry (B474, Micronal, São Paulo, Brazil). The nanocapsule size was measured by different techniques, including laser diffraction (Mastersizer 2000, Nano ZS; Malvern Instruments, Malvern, UK), dynamic light scattering (Zetasizer Nano ZS; Malvern Instruments), and particle tracking (Nanosight LM 10), by dilution of nanocapsules in bidistilled water. For the determination of the zeta potential, the nanocapsules were diluted in $\mathrm{NaCl}$ solution $(10 \mathrm{mM})$ and electrophoretic mobility was analyzed (Zetasizer, Nano ZS).

\section{Production of chitosan gels}

First, different formulations with increasing chitosan concentrations $(1.5 \%, 2 \%, 2.5 \%$, and $3 \%$ weight/weight $[\mathrm{w} / \mathrm{w}])$ were produced to determine the most suitable chitosan gel for vaginal administration. The hydrogels were prepared by manual mixing of chitosan, lactic acid $(115 \mu \mathrm{L})$, and bidistilled water or nanocapsule suspension without the fluorescent marker $(10 \mathrm{~mL})$. The formulations were named CH-RS (chitosan gel containing Eudragit RS 100 nanocapsules), $\mathrm{CH}-\mathrm{S}$ (chitosan gel containing Eudragit S 100 nanocapsules), and $\mathrm{CH}$ (chitosan gel prepared with water).

After determining the most suitable gel, nanocapsules containing NR were loaded in the selected chitosan gel and the formulations were named $\mathrm{CH}-\mathrm{RS}-\mathrm{NR}$ (chitosan gel containing Eudragit RS 100 nanocapsules) and CH-S-NR (chitosan gel containing Eudragit S 100 nanocapsules). For the gel obtained with water instead of nanocapsules (CH-NR), $330 \mu \mathrm{L}$ of the mixture of NR and capric/caprylic triglycerides $(2.5 \mathrm{mg} / \mathrm{mL})$ was added during the production of $10 \mathrm{~g}$ of the formulation. Therefore, at the final stage, all of the gels had the same concentration of NR $(0.0825 \mathrm{mg} / \mathrm{g})$.

\section{Characterization of chitosan gels}

Gels at increasing concentrations of chitosan (from 1.5\% to $3 \%$ ) were evaluated in terms of $\mathrm{pH}$ values and rheological properties to identify the most suitable gel for vaginal application. $\mathrm{pH}$ values were measured by potentiometry (B474, Micronal) after dilution of the formulation in ultrapure water (1:10 weight:volume [w:v]). Each formulation was subjected to rheological characterization by rotational rheometry (Rheostress 600; Haake Technik, Vreden, Germany) at $25^{\circ} \mathrm{C}$ using a cone plate combination (C35/1: $35 \mathrm{~mm}$ diameter and $1^{\circ}$ angle) as the measuring system. The rheological measurements were performed after 3 minutes of rest time. The apparent viscosity was determined at $200 \mathrm{~s}^{-1}$. Dynamic oscillatory tests were carried out in the linear viscoelastic range at $25^{\circ} \mathrm{C}$; a constant shear stress value (previously determined in the linear viscoelastic region) was applied to the sample, and the viscoelastic response of the sample was recorded and expressed by the conservative or elastic modulus $\mathrm{G}^{\prime}$ and viscous or dissipative $G^{\prime \prime}$ modulus. $G^{\prime}$ and $G^{\prime \prime}$ were recorded at $10 \mathrm{~Hz}$. The loss tangent $\left(\operatorname{tg} \delta\right.$ ) was calculated as the $G^{\prime \prime}$ to $G^{\prime}$ ratio.

\section{In vitro studies using vaginal mucosa}

After the most suitable chitosan concentration was selected, based on the viscosity, elasticity and $\mathrm{pH}$, gels containing nanocapsules or water were analyzed in terms of in vitro mucoadhesion, washability, and NR penetration into the vaginal mucosa. For the mucoadhesion experiment, previously frozen $\left(-20^{\circ} \mathrm{C}\right)$ vaginal mucosa was used while, for the washability and penetration experiments, fresh mucosa was used to maintain the properties and viability of the tissue. To correctly simulate the vaginal environment, the washing liquid selected was phosphate buffered saline $\mathrm{pH} 4.5$ maintained at $37^{\circ} \mathrm{C}$.

\section{Mucoadhesion measurements}

The samples ( $\mathrm{CH}, \mathrm{CH}-\mathrm{RS}$, and $\mathrm{CH}-\mathrm{S})$ were subjected to mucoadhesion measurements by means of a tensile stress tester (TA.XTplus Texture Analyzer; Stable Microsystem, Godalming, UK) equipped with a $1 \mathrm{~kg}$ load cell and a measuring system. The measuring system consists of a probe and a support, both made of Teflon. The support consists of two cylinders; the lower cylinder serves as a base for the biological substrate, while the upper cylinder has a circular hole in the center $(\varnothing: 14 \mathrm{~mm})$, which allows the probe to enter.

Each sample of mucosa was wet with $40 \mu \mathrm{L}$ of acetate buffer, $\mathrm{pH} 4.5$, in order to simulate the vaginal fluid. Forty microliters of each sample was layered on a filter paper disc ( $: 10 \mathrm{~mm}$ ) that was fixed to the cylindrical probe with biadhesive tape. After a predetermined time (180 seconds), 
the cylindrical probe was put in contact with the sample by applying a 2,500 $\mathrm{mN}$ preload for 3 minutes. The probe was lifted at $2.5 \mathrm{~mm} / \mathrm{min}$ until complete separation of the mucoadhesion interface was achieved.

The experimental parameters were chosen to enable accurate and reproducible results as well as the best differentiation between the various samples. In particular, the choice was made on the basis of preliminary tests at various detachment rates and preload values. The force of detachment $(\mathrm{mN})$ and the force of detachment as a function of displacement (work of adhesion $[\mathrm{mN} \cdot \mathrm{mm}]$ ) were determined for all formulations.

\section{Washability test}

The samples (CH-NR, CH-RS-NR, and CH-S-NR) were subjected to washability measurements by means of a Franz diffusion cell (Permeager; Hellertown, PA, USA) with a modified donor chamber as previously described. ${ }^{29,30}$ Briefly, in the donor chamber, a stream of buffer ( $\mathrm{pH} 4.5)$ was fluxed between two holes. The flux was set at $0.2 \mathrm{~mL} / \mathrm{min}$ with a high-performance liquid chromatography pump (HPLC pump 420; Kontron AG, Eching, Germany). The outgoing buffer was collected in a beaker with continuous stirring. The pig vaginal mucosa was layered on a Parafilm membrane (impermeable to fluids) and divided between the donor and acceptor chambers of a Franz cell. Distilled water was used as the receptor phase to maintain the vaginal mucosa at $37^{\circ} \mathrm{C}$. Each sample (100 mg) was spread on the vaginal mucosa, and $\mathrm{pH} 4.5$ buffer was fluxed $\left(37^{\circ} \mathrm{C}\right)$ over the sample to simulate the removal action of vaginal fluid. The $\mathrm{pH} 4.5$ buffer exiting the donor chamber was collected in different beakers, filling one beaker per hour up to 6 hours and obtaining six fractions. The beakers were frozen and the content was lyophilized for 24 hours (Heto Dryer; Analitical De-Mori, Milan, Italy). Then, each pellet was reconstituted in $500 \mu \mathrm{L}$ of acetone to solubilize the Eudragit polymers. The samples were then diluted with isoamyl acetate to a suitable concentration. Each sample was assayed for NR using a spectrofluorimetric method. The excitation ( $\lambda$ ex $)$ and emission ( $\lambda$ em) wavelengths applied to all samples were $517 \mathrm{~nm}$ and $587 \mathrm{~nm}$, respectively. The method was linear in the range from $6.25 \mathrm{ng} / \mathrm{mL}$ to $100 \mathrm{ng} / \mathrm{mL}$ with an $R^{2}$ always higher than 0.9995 .

After the washability experiments, the vaginal tissues were rinsed twice in saline solution to eliminate sample residues and were frozen in liquid nitrogen. Three samples were processed to evaluate the amount of NR that penetrated into the tissue as a function of the tissue depth, and three samples were subjected to confocal laser scanning microscopy (CLSM) analysis.
NR penetration into porcine vaginal mucosa

A cryostat (working temperature $-20^{\circ} \mathrm{C}$ ) (Leica CM1510; Leica Microsystem, Wetzlar, Italy) was used to cut horizontal slices $50 \mu \mathrm{m}$ in thickness. Four slices were collected in Eppendorf microtubes. The amount of NR that penetrated into the different slices was extracted by adding $300 \mu \mathrm{L}$ of isoamyl acetate and $50 \mu \mathrm{L}$ of acetone. The samples were maintained while shaking for 15 minutes at room temperature and subjected to centrifugation $(5,000 \times g)$ to separate the tissue residues from the supernatant.

A calibration curve was prepared by processing the samples as described above. The method was linear in the range from $6.25 \mathrm{ng} / \mathrm{mL}$ to $100 \mathrm{ng} / \mathrm{mL}$ with an $R^{2}$ always higher than 0.9995 .

\section{CLSM analysis of NR penetration into porcine vaginal mucosa}

Vaginal mucosa tissues subjected to washability experiments were rinsed twice with phosphate buffer, $\mathrm{pH} 7.4$ (USP 35) and frozen in liquid nitrogen after inclusion in Optimal Cutting Temperature compound (Leica Microsystem). The frozen samples were stored at $-80^{\circ} \mathrm{C}$ until the analysis.

NR internalized in the vaginal tissues was analyzed using CLSM. Each frozen vaginal sample was cut perpendicularly to the mucosa surface in $25 \mu \mathrm{m}$-thick slices using a cryostat (working temperature $-20^{\circ} \mathrm{C}$ ). After the deposition on a microscope slide, each slice was dehydrated for 12 hours and dipped in acetone for fixing. The nuclei present in the tissue slices were stained using 4',6-diamidino-2-phenylindole (DAPI; Sigma-Aldrich) at 1:100,000. The tissue slices on microscope slides were dipped in DAPI solution for 2 minutes and washed in phosphate buffer, $\mathrm{pH} 7.4$, to eliminate the staining excess that did not react with the DNA.

After drying the tissue slices at room temperature for 12 hours, polyvinyl alcohol mounting medium with DABCO antifading (BioChemika, Fluka, Italy) - a mixture of tris (hydroxymethyl) aminomethane/tris (hydroxymethyl) aminomethane hydrochloride, 22,000 Da polyvinyl alcohol, anhydrous glycerol, and 1,4-diazabicyclo[2,2,2] octane - was spread on a microscope slide and a cover glass was applied.

The CLSM analysis was performed by using $\lambda$ ex $=549 \mathrm{~nm}$ and $\lambda \mathrm{em}=628 \mathrm{~nm}$ for the visualization of $\mathrm{NR}$ and $\lambda$ ex $=340 \mathrm{~nm}$ and $\lambda \mathrm{em}=488 \mathrm{~nm}$ for the visualization of DAPI. The acquired images were processed by means of specific software (Leica Microsystem). 


\section{Statistical analyses}

Significant differences were determined by means of analysis of variance followed by Tukey's post hoc test for multiple comparisons. Differences between groups were considered to be significant at $P<0.05$. Analyses were performed with SPSS Statistics $17.0^{\circledR}$ software (SPSS Inc., Chicago, IL, USA).

\section{Results and discussion Properties of nanocapsules}

Table 1 shows the nanocapsule sizes measured by different techniques. All of the particles had nanometer diameters (approximately $200 \mathrm{~nm}$ ) and adequate homogeneity of size distribution (polydispersity index $[\mathrm{PDI}] \leq 0.2$ ). The incorporation of NR and the type of polymer used did not significantly change the size of the nanocapsules. Through such analyses, it was also possible to determine the concentration of nanoparticles in the aqueous suspension (particles per $\mathrm{mL}$ ). Similar values were observed for all of the formulations $\left(\mathrm{NC}-\mathrm{RS}=11.83 \pm 2.5 \times 10^{12}\right.$, NC-S $=7.66 \pm 0.67 \times 10^{12}$, NC-RS-NR $=9.0 \pm 2.4 \times 10^{12}, \mathrm{NC}-\mathrm{S}-\mathrm{NR}=8.9 \pm 1.0 \times 10^{12}$ ). This is an important parameter for an accurate comparison of formulations.

Regarding the zeta potential, the nanocapsules produced with Eudragit RS 100 presented positive values $(+13.6 \pm 0.8 \mathrm{mV}$ for NC-RS and $+10.45 \pm 0.9 \mathrm{mV}$ for $\mathrm{NC}-$ RS-NR), while nanocapsules produced with Eudragit S 100 presented negative values $(-12.93 \pm 0.6 \mathrm{mV}$ for NC-S and $-10.78 \pm 1.15 \mathrm{mV}$ for NC-S-NR), as previously described. ${ }^{31}$ The positive values observed for formulations NC-RS and NC-RS-NR are due to the trimethyl-ammoniummethyl-methacrylate present in Eudragit RS 100.

The NC-S and NC-S-NR formulations had negative zeta potentials due to the methacrylic acid present in Eudragit $\mathrm{S} 100$. Charge differences in the zeta potential of nanocapsules were studied to determine the effect of a lipophilic substance on vaginal application. The nanocapsule suspensions presented $\mathrm{pH}$ values of $6.1 \pm 0.1$ (NC-RS), $5.3 \pm 0.2$ (NC-S), $6.2 \pm 0.1$ (NC-RS-NR), and 5.1 \pm 0.1 (NC-S-NR).

\section{Selection of the most suitable hydrogel for vaginal application}

Rheology and $\mathrm{pH}$ measurements were applied to select the most suitable chitosan concentration for vaginal application. The gels presented $\mathrm{pH}$ values varying from 3.8 (1.5\% chitosan) to 5.2 (3\% chitosan) due to the presence of lactic acid, as already described by Contri et al. ${ }^{24}$ Except for the formulation produced with $3 \%$ chitosan, all of the gels were considered suitable for vaginal administration because their $\mathrm{pH}$ values were compatible with the $\mathrm{pH}$ at the site of application. The $\mathrm{pH}$ of vaginal mucosa is between 3.5 and 4.5 , maintained by the lactobacilli that convert the glycogen of the epithelium into lactic acid. ${ }^{2}$

Regarding the rheological properties, all chitosan gels presented a decrease in viscosity with an increase in shear rate, indicating that they are non-Newtonian fluids with pseudoplastic behavior (Figure 1). Increases in the chitosan concentrations followed by increases in the viscosity were also observed visually, as shown by Perioli et $\mathrm{al}^{3}$ and Contri et al. ${ }^{24}$

For a better comparison between formulations, the rheological parameters were obtained at a shear rate of $200 \mathrm{~s}^{-1}$ (viscosity) and a frequency of $10 \mathrm{~Hz}$ (oscillatory rheology data) at $25^{\circ} \mathrm{C}$ (Table 2). The following two variables were studied: 1) addition of nanocapsules (NC-RS or NC-S) to the chitosan gels; and 2) increase of chitosan concentration (from $1.5 \%$ to $3 \%$ ). Table 2 shows that the addition of nanocapsules increased the viscosity of gels at all studied concentrations $(P<0.001)$. Comparing the two types of gels containing nanocapsules $(\mathrm{CH}-\mathrm{RS}$ and $\mathrm{CH}-\mathrm{S}$ ), the viscosities were similar except at a chitosan concentration of $3 \%$, in which case the gel containing

Table I Size characterization of nanocapsule suspensions by different techniques

\begin{tabular}{|c|c|c|c|c|c|c|}
\hline \multirow{2}{*}{$\begin{array}{l}\text { Nanocapsule } \\
\text { suspension }\end{array}$} & \multicolumn{3}{|c|}{ Light diffraction* } & \multicolumn{2}{|c|}{ Dynamic light scattering** } & \multirow{2}{*}{$\begin{array}{l}\text { Particle } \\
\text { tracking } \\
(\mathrm{nm})\end{array}$} \\
\hline & d (0.5) (nm) & $D[4.3](n m)$ & span & d-average $(\mathrm{nm})$ & PDI & \\
\hline NC-RS & $158.6 \pm 5.8$ & $169.3 \pm 4.7$ & $0.4 \pm 0.0$ & $168.5 \pm 18.7$ & $0.1 \pm 0.0$ & $146 \pm 4.36$ \\
\hline NC-S & $171.8 \pm 16.5$ & $286.3 \pm 17.5$ & $0.8 \pm 0.1$ & $204.9 \pm 6.0$ & $0.2 \pm 0.0$ & $206 \pm 16.2$ \\
\hline NC-RS-NR & $122.0 \pm 37.0$ & $185.3 \pm 33.3$ & $0.9 \pm 0.1$ & $196.5 \pm 21.0$ & $0.1 \pm 0.0$ & $180.7 \pm 18.5$ \\
\hline NC-S-NR & $144.2 \pm 24.5$ & $279.0 \pm 17.0$ & $1.0 \pm 0.2$ & $185.7 \pm 7.9$ & $0.1 \pm 0.0$ & $201.7 \pm 9.0$ \\
\hline
\end{tabular}

Notes: Values displayed as mean \pm standard deviation; $n=3$. *Values were obtained by analysis of number. **Values were obtained by analysis of intensity.

Abbreviations: NC-RS, Eudragit ${ }^{\circledR}$ RS 100 nanocapsules; NC-S, Eudragit ${ }^{\circledR}$ S 100 nanocapsules; NC-RS-NR, Nile red Eudragit RS I00 nanocapsules; NC-S-NR, Nile red Eudragit S 100 nanocapsules; d, diameter for light diffraction; D, average diameter for light diffraction; d-average, average diameter for dynamic light scattering; PDI, polydispersity index. 

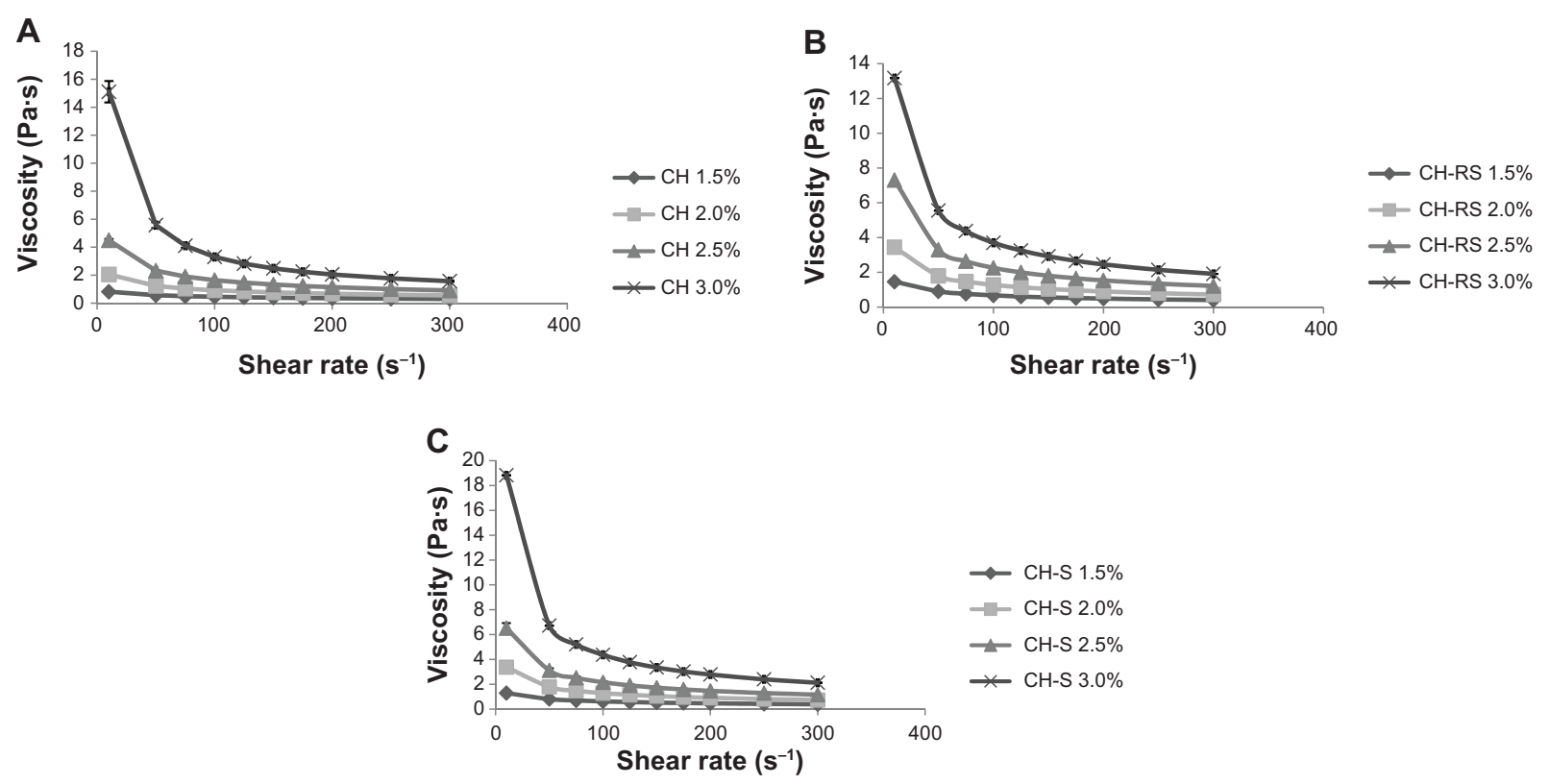

Figure I Viscosity as a function of shear rate graphs.

Notes: (A) Chitosan hydrogels, (B) chitosan hydrogels with Eudragit ${ }^{\circledR}$ RS 100 nanocapsules, and (C) chitosan hydrogels with Eudragit ${ }^{\circledR}$ S I00 nanocapsules (Evonik Industries AG, Essen, Germany). Mean \pm standard deviation, $n=3$.

Abbreviations: $\mathrm{CH}$, chitosan hydrogel; $\mathrm{CH}-\mathrm{RS}$, chitosan hydrogel with Eudragit RS 100 nanocapsules; $\mathrm{CH}-\mathrm{S}$, chitosan hydrogel with Eudragit $\mathrm{S}$ I00 nanocapsules.

anionic nanocapsules presented higher viscosity. This result might be related to a higher viscosity of the formulation obtained with NC-S due to the use of sorbitan monostearate or the polymer characteristics. This result could also be related to the increased interaction between the anionic particles and the chitosan polymeric chains. In terms of the rigidity/elasticity of the gels, Table 2 shows that the incorporation of nanocapsules did not clearly influence the oscillatory parameters at the frequency studied. More rigid structures were obtained when NC-S was incorporated into the $3 \%$ chitosan gel $(P<0.001)$. The higher rigidity could also be related to the interaction between chitosan chains and anionic nanocapsules, as described for the viscosity. The difference in measured rigidity was also observed visually; the formulation composed of NC-S presented a glue-like aspect of low spreadability.

For all of the formulations studied (different chitosan concentrations), Table 2 shows that a decrease in $\operatorname{tg} \delta$ values occurred with increased chitosan concentrations. These findings indicate that the higher the chitosan concentration, the higher the rigidity of the gel. Such behavior is desirable to retain the formulation at the site of application. This helps to avoid the flow of product out of the desired area, leading to a possible increase of the formulation efficacy.

Table 2 Rheological parameters at $25^{\circ} \mathrm{C}$ of formulations at different chitosan concentrations with and without polymeric nanocapsules

\begin{tabular}{|c|c|c|c|c|}
\hline Hydrogel & $\begin{array}{l}\text { Viscosity at } \\
200 \mathrm{~s}^{-1} \text { (Pa.s) }\end{array}$ & $\begin{array}{l}\text { G' (Pa) at } \\
10 \mathrm{~Hz}\end{array}$ & $\begin{array}{l}\text { G" (Pa) at } \\
10 \mathrm{~Hz}\end{array}$ & $\begin{array}{l}\operatorname{tg} \delta \text { at } \\
10 \mathrm{~Hz}\end{array}$ \\
\hline $\mathrm{CH} .5 \%$ & $0.35 \pm 0.01$ & $15.14 \pm 2.46$ & $22.79 \pm 3.69$ & $1.56 \pm 0.54$ \\
\hline $\mathrm{CH} 2.0 \%$ & $0.66 \pm 0.01$ & $56.92 \pm 13.53$ & $73.78 \pm 7.47$ & $1.33 \pm 0.20$ \\
\hline $\mathrm{CH} 2.5 \%$ & $1.14 \pm 0.01$ & $106.91 \pm 13.21$ & $113.06 \pm 7.15$ & $1.06 \pm 0.07$ \\
\hline $\mathrm{CH} 3.0 \%$ & $2.18 \pm 0.23$ & $208.53 \pm 6.99$ & $174.61 \pm 7.16$ & $0.84 \pm 0.0 \mathrm{I}$ \\
\hline CH-RS I.5\% & $0.49 \pm 0.05$ & $29.12 \pm 1.86$ & $43.54 \pm 2.86$ & $1.50 \pm 0.08$ \\
\hline CH-RS $2.0 \%$ & $0.90 \pm 0.04$ & $63.37 \pm 4.94$ & $86.43 \pm 4.01$ & $1.37 \pm 0.04$ \\
\hline $\mathrm{CH}-\mathrm{RS} 2.5 \%$ & $1.53 \pm 0.03$ & $127.25 \pm 0.02$ & $148.46 \pm 0.43$ & $1.26 \pm 0.16$ \\
\hline CH-RS $3.0 \%$ & $2.45 \pm 0.01$ & $235.5 \pm 15.27$ & $248.35 \pm 14.34$ & $1.05 \pm 0.0 \mathrm{I}$ \\
\hline CH-S I.5\% & $0.46 \pm 0.01$ & $31.15 \pm 2.14$ & $42.70 \pm 3.26$ & $1.30 \pm 0.13$ \\
\hline CH-S $2.0 \%$ & $0.90 \pm 0.00$ & $64.44 \pm 0.27$ & $79.63 \pm 0.51$ & $1.19 \pm 0.08$ \\
\hline $\mathrm{CH}-\mathrm{S} 2.5 \%$ & $1.46 \pm 0.08$ & $|37.3 \pm| 3.0 \mid$ & $|4| .09 \pm 8.73$ & $0.98 \pm 0.09$ \\
\hline CH-S 3.0\% & $2.78 \pm 0.08$ & $338.23 \pm 37.05$ & $254.75 \pm 21.10$ & $0.75 \pm 0.02$ \\
\hline
\end{tabular}

Notes: Values displayed as mean \pm standard deviation; $n=3$. Eudragit ${ }^{\circledR}$ RS 100 and Eudragit ${ }^{\circledR}$ S 100 (Evonik Industries AG, Essen, Germany).

Abbreviations: $\mathrm{CH}$, chitosan hydrogel; $\mathrm{CH}$-RS, chitosan hydrogel with Eudragit RS 100 nanocapsules; $\mathrm{CH}-\mathrm{S}$, chitosan hydrogel with Eudragit S I00 nanocapsules; G', storage modulus; G", loss modulus; tg $\delta$, loss tangent parameter calculated from the ratio between G" and G'. 
Given that it had the highest values of measured viscosity and highest elasticity of gels (lower values of $\operatorname{tg} \delta$ ), the $3.0 \% \mathrm{w} / \mathrm{w}$ chitosan gel was chosen as the most suitable for vaginal delivery. However, this formulation presented some disadvantages. The $\mathrm{pH}$ values were higher than 4.5, which could disturb the microbial environment. Additionally, the visual viscosity was inadequate (too high), and a difficulty in application on the mucosa was observed during pretests. Such properties could compromise the removal from the applicator and the correct application to the tissue. Therefore, the gels composed of $2.5 \% \mathrm{w} / \mathrm{w}$ chitosan (CH, CH-RS, and CH-S), which presented a $\mathrm{pH}$ of 4.5 and suitable rheology properties, were chosen for further experiments using vaginal mucosa (mucoadhesion, washability, and penetration).

\section{In vitro adhesion to vaginal mucosa}

In vitro adhesion of the formulation to the vaginal mucosa was studied by means of mucoadhesion experiments using a tensile stress tester and by analyzing the washability profiles. Gels with $2.5 \% \mathrm{w} / \mathrm{w}$ chitosan with and without nanocapsules were evaluated (CH-NR, CH-RS-NR, and CH-S-NR).

For the tensile stress measurements, the force, displacement distance, and work needed to detach the hydrogels from the vaginal mucosa were determined, as shown in Figure 2. This figure shows the average force necessary to separate the formulation from the mucosa and

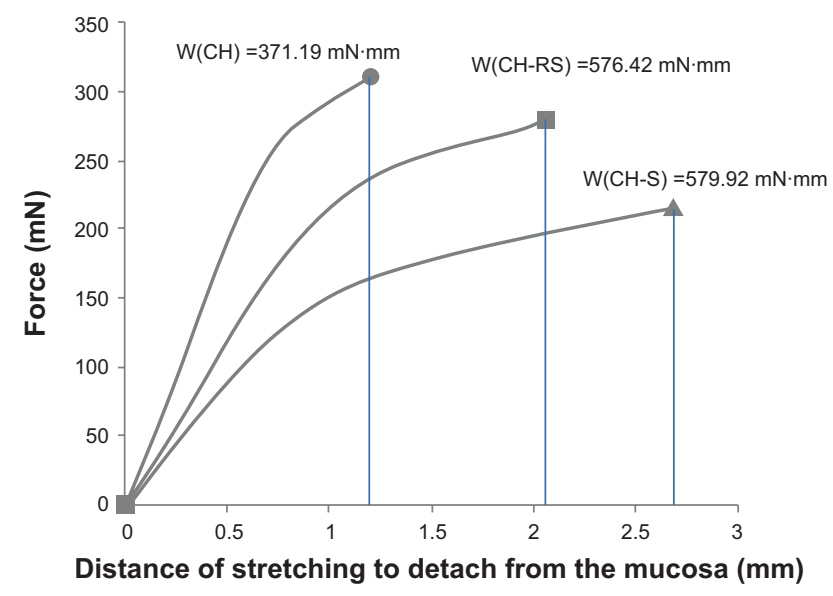

Figure 2 Mucoadhesion of chitosan hydrogels ( $2.5 \%$ chitosan) determined by the force of detachment and the distance of stretching to detach from the mucosa (mean, $\mathrm{n}=6$ ).

Notes: Eudragit ${ }^{\circledR}$ RS 100 and Eudragit ${ }^{\circledR}$ S I00 (Evonik Industries AG, Essen, Germany). The area under the curve represents the work (ie, the product of force and distance) necessary to detach a formulation from the mucosa. Such work values $[\mathrm{W}(\mathrm{CH})$; $\mathrm{W}(\mathrm{CH}-\mathrm{RS}) ; \mathrm{W}(\mathrm{CH}-\mathrm{S})]$ are a representation of the mucoadhesive properties of each formulation.

Abbreviations: $\mathrm{CH}$, chitosan hydrogel; $\mathrm{CH}-\mathrm{RS}$, chitosan hydrogel with Eudragit RS 100 nanocapsules; $\mathrm{CH}-\mathrm{S}$, chitosan hydrogel with Eudragit S 100 nanocapsules. the displacement distance achieved for the formulation to detach from the mucosa. The area under the curve (Figure 2) represents the work (ie, the product of force and distance) necessary to detach a formulation from the mucosa. Such work values [W(CH); W(CH-RS); W(CH-S)] are a representation of the mucoadhesive properties of each formulation. ${ }^{1}$

Figure 2 shows that the $\mathrm{CH}$ formulation required a higher average effort to achieve detachment, while it traveled a shorter stretching distance to detach from the mucosa. In contrast, the $\mathrm{CH}-\mathrm{S}$ formulation required lower average effort but reached higher stretching distances for the formulation to detach from the tissue. Formulation $\mathrm{CH}-\mathrm{RS}$ presented intermediate behavior. Considering the absolute values of work for each formulation (Figure 2), significant differences were found between the samples $(P=0.033)$. In this sense, hydrogels with nanocapsules (CH-RS and $\mathrm{CH}-\mathrm{S}$ ) showed higher values of work for detachment compared with the hydrogels without nanocapsules $(\mathrm{CH})$, while no differences were found due to the type of nanocapsule incorporated in the gel (CH-RS and $\mathrm{CH}-\mathrm{S}$ ). Consequently, the gels with nanocapsules (CH-RS and $\mathrm{CH}-\mathrm{S}$ ) have the potential to stay in contact with the mucosa for a longer time. Such results might be related to the higher viscosity due to the incorporation of nanocapsules and to the structural changes that the nanocapsules induce in the gel network. ${ }^{32}$

Looking at Figure 2, in terms of the material properties of the formulations, it can be concluded that hydrogels containing nanocapsules (CH-RS and $\mathrm{CH}-\mathrm{S}$ ) presented a ductile behavior because a lower force was necessary to separate the formulation and because greater stretching distances were observed. In contrast, chitosan gel without nanocapsules appeared to be fragile; stronger forces were applied and shorter stretching distances were traveled until detachment.

Additionally, hydrogels with and without polymeric nanocapsules and the fluorescent marker NR (CH-RS-NR, $\mathrm{CH}-\mathrm{S}-\mathrm{NR}, \mathrm{CH}-\mathrm{NR}$ ) were tested in the washability experiments. The experiment was performed for 6 hours, which is the average time that the formulations, when applied in the vaginal route of administration, should be kept in contact with the mucosa to present the desired effect. Figure 3 shows the washability profiles (percentage of NR washed away as a function of time). No significant differences were observed among formulations (analysis of variance, $P=0.107$ ) for all of the studied times. One possible reason for this is that, when time is considered in the analysis, the most relevant factor to be considered is the type of vehicle that will be washed, ie, the chitosan. Because the three formulations were derived from 


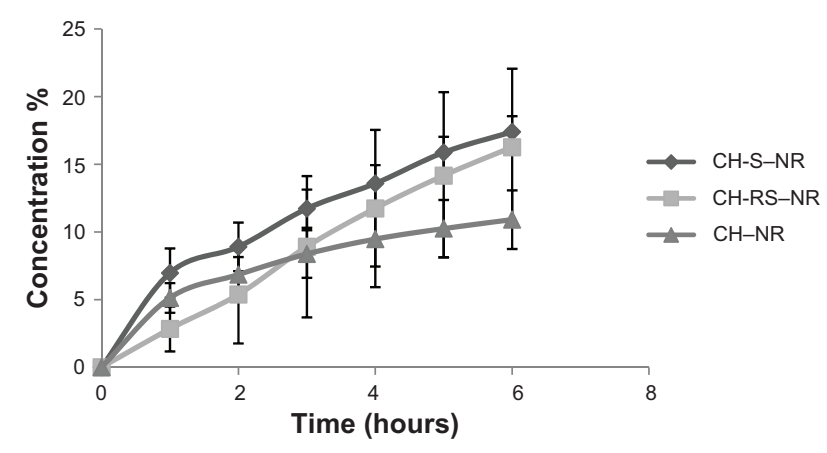

Figure 3 Nile red washability profiles after incorporation into chitosan hydrogels (mean \pm standard error, $\mathrm{n}=3$ ).

Notes: $2.5 \%$ chitosan. Eudragit ${ }^{\circledR}$ RS 100 and Eudragit ${ }^{\circledR}$ S 100 (Evonik Industries AG, Essen, Germany).

Abbreviations: $\mathrm{CH}$, chitosan hydrogel; $\mathrm{CH}-\mathrm{RS}$, chitosan hydrogel with Eudragit RS 100 nanocapsules; $\mathrm{CH}-\mathrm{S}$, chitosan hydrogel with Eudragit S 100 nanocapsules; NR, Nile red.

the same polymer and had the same concentration $(2.5 \% \mathrm{w} / \mathrm{w})$, no differences between formulations were detected. In this sense, formulations containing nanocapsules were washed in the same way as those only composed of chitosan and NR.

\section{In vitro penetration into vaginal mucosa}

The in vitro penetration into vaginal mucosa was determined by the following two techniques: 1) quantification of the fluorescence as a function of the mucosa depth after extraction from the tissue; and 2) CLSM. In both experiments, the drug penetration was determined on vaginal substrates subjected to the washability test and loaded with NR.

Table 3 shows the total amounts of NR that penetrated into the tissue, and the profiles of the amount of NR $(\mu \mathrm{g})$ that penetrated as a function of vaginal tissue depth are shown in Figure 4. According to the results shown in Table 3 and Figure 4, when NR was encapsulated into nanocapsules, the penetration amount was higher. Figure 4 shows that the NR in $\mathrm{CH}-\mathrm{RS}-\mathrm{NR}$ penetrated threefold deeper, while that of $\mathrm{CH}-\mathrm{S}-\mathrm{NR}$ penetrated almost twofold deeper. The analysis of NR penetration profiles revealed the different behavior of

Table 3 Amounts of NR that penetrated into vaginal tissue (mean \pm standard deviation, $\mathrm{n}=3$ ) and enhancement ratio calculated as the ratio between nanoencapsulated NR formulation and NR in chitosan gel

\begin{tabular}{lll}
\hline Formulation & $\begin{array}{l}\text { Total amount of NR } \\
\text { penetrated into the tissue }(\mu \mathbf{g})\end{array}$ & $\begin{array}{l}\text { Enhancement } \\
\text { ratio }\end{array}$ \\
\hline $\mathrm{CH}-\mathrm{S}-\mathrm{NR}$ & $0.4 \mathrm{I} \pm 0.08$ & 2.05 \\
$\mathrm{CH}-\mathrm{RS}-\mathrm{NR}$ & $0.60 \pm 0.04$ & 3 \\
$\mathrm{CH}-\mathrm{NR}$ & $0.20 \pm 0.03$ & - \\
\hline
\end{tabular}

Abbreviations: $\mathrm{CH}$, chitosan hydrogel; $\mathrm{CH}-\mathrm{RS}$, chitosan hydrogel with Eudragit ${ }^{\circledR}$ RS 100 nanocapsules; CH-S, chitosan hydrogel with Eudragit ${ }^{\circledR}$ S 100 nanocapsules; NR, Nile red. the formulations; CH-NR presented almost a linear pattern with homogeneous distribution of NR penetration into the tissue. The formulations in which NR was nanoencapsulated presented different patterns, in particular at the depths ranging between 2,200 and 3,800 $\mu \mathrm{m}$, where significant differences among formulations were found $(P<0.001)$. CH-RS-NR presented higher values than the other formulations tested at the mentioned depth (from 2,200 $\mu \mathrm{m}$ to 3,800 $\mu \mathrm{m}$ ). At 2,600 $\mu \mathrm{m}, 3,400 \mu \mathrm{m}$, and 3,800 $\mu \mathrm{m}, \mathrm{CH}-\mathrm{S}-\mathrm{NR}$ also significantly differed from $\mathrm{CH}-\mathrm{NR}$.

The nanoencapsulation of the NR increased its penetration into the mucosa, probably due to different factors: 1) the small size, as observed previously by Torchilin, ${ }^{33}$ which allow them to be easily transported through barriers, such as the mucus in the mucosal epithelium by passive or active transport - Mallipeddi and Rohan ${ }^{34}$ also observed that, due to their dimension, nanoparticles can penetrate through and into cells, delivering drugs without suffering extracellular degradation; 2) the bioadhesion of the nanostructures, as observed previously by Contri et $\mathrm{al}^{17}$ which contributes to the increase in the residence time of the drug on the tissue, leading to an enhancement of the substance penetration; 3 ) the controlled release property, which can prolong the release and increase the contact time between the drug and the mucosa. ${ }^{17}$ Also, the type of polymer composing the particle shell, represented in this work by Eudragit RS 100 and Eudragit S 100, may have played an important role on the penetration into the mucosa. Nanocapsules based on Eudragit RS 100 showed NR accumulation at approximately 2,500-3,000 nm with a sharp peak, while nanocapsules based on Eudragit S 100 accumulated at depths ranging from $1,000 \mathrm{~nm}$ to $2,400 \mathrm{~nm}$, with a lower and smoother peak than that observed for Eudragit RS 100 nanocapsules. The different behavior is likely related to the different ionic character of the two polymers; Eudragit RS 100 is cationic, and Eudragit S 100 is anionic. The cationic charge of RS nanoparticles could allow CH-RS-NR to develop an intimate contact with the negatively-charged mucosal surface. Therefore, the nanoencapsulation of a hydrophobic active ingredient, especially in positively charged nanocapsules, could increase the penetration into the vaginal mucosa, improving the drug's efficacy. Moreover, an increase in penetration could also enable a reduction of dose.

Figure 5 shows the CLSM images of the vaginal mucosa after application of the different chitosan formulations and after subjection to the washability experiments. Figure 5A represents the mucosa without the application of any formulation. Figure 5B, C, and D were obtained after application 


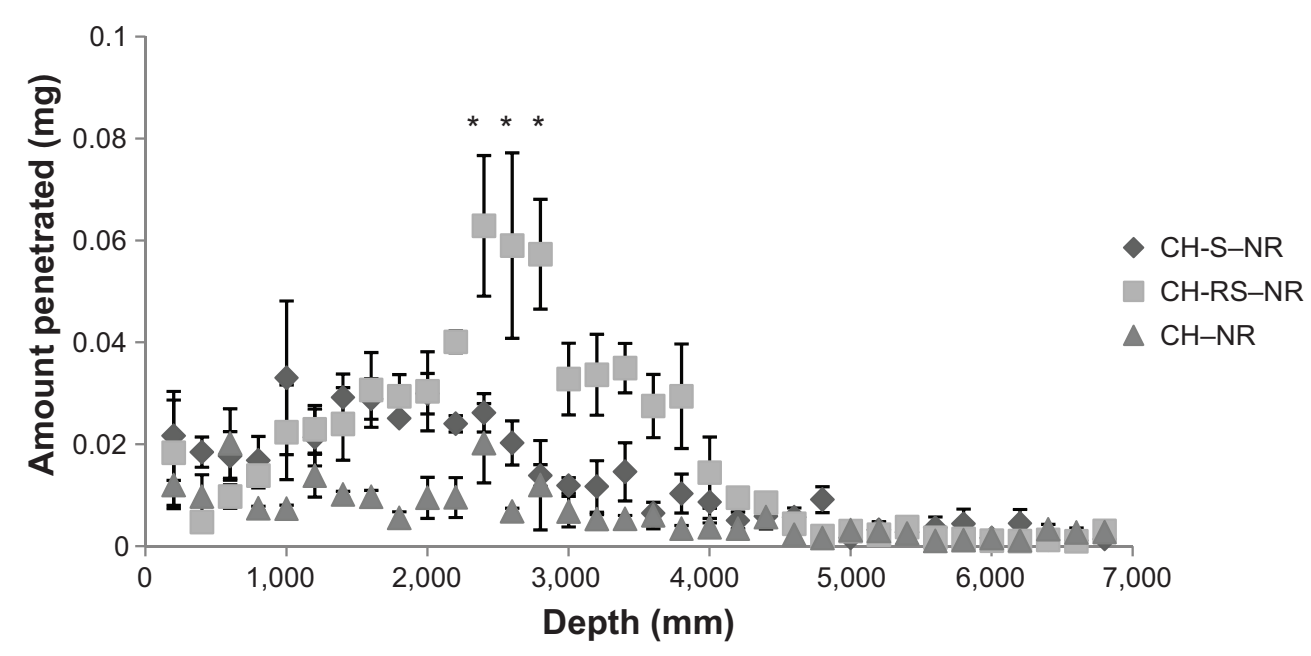

Figure 4 Quantification of Nile red accumulation into vaginal mucosa as a function of mucosa depth, after incorporation into chitosan hydrogels (mean \pm standard error, $\mathrm{n}=3$ ).

Notes: $2.5 \%$ chitosan. $* P<0.001$. Eudragit ${ }^{\circledR}$ RS 100 and Eudragit ${ }^{\circledR}$ S 100 (Evonik Industries AG, Essen, Germany).

Abbreviations: $\mathrm{CH}$, chitosan hydrogel; $\mathrm{CH}-\mathrm{RS}$, chitosan hydrogel with Eudragit RS 100 nanocapsules; $\mathrm{CH}-\mathrm{S}$, chitosan hydrogel with Eudragit $\mathrm{S}$ I00 nanocapsules; NR, Nile red.
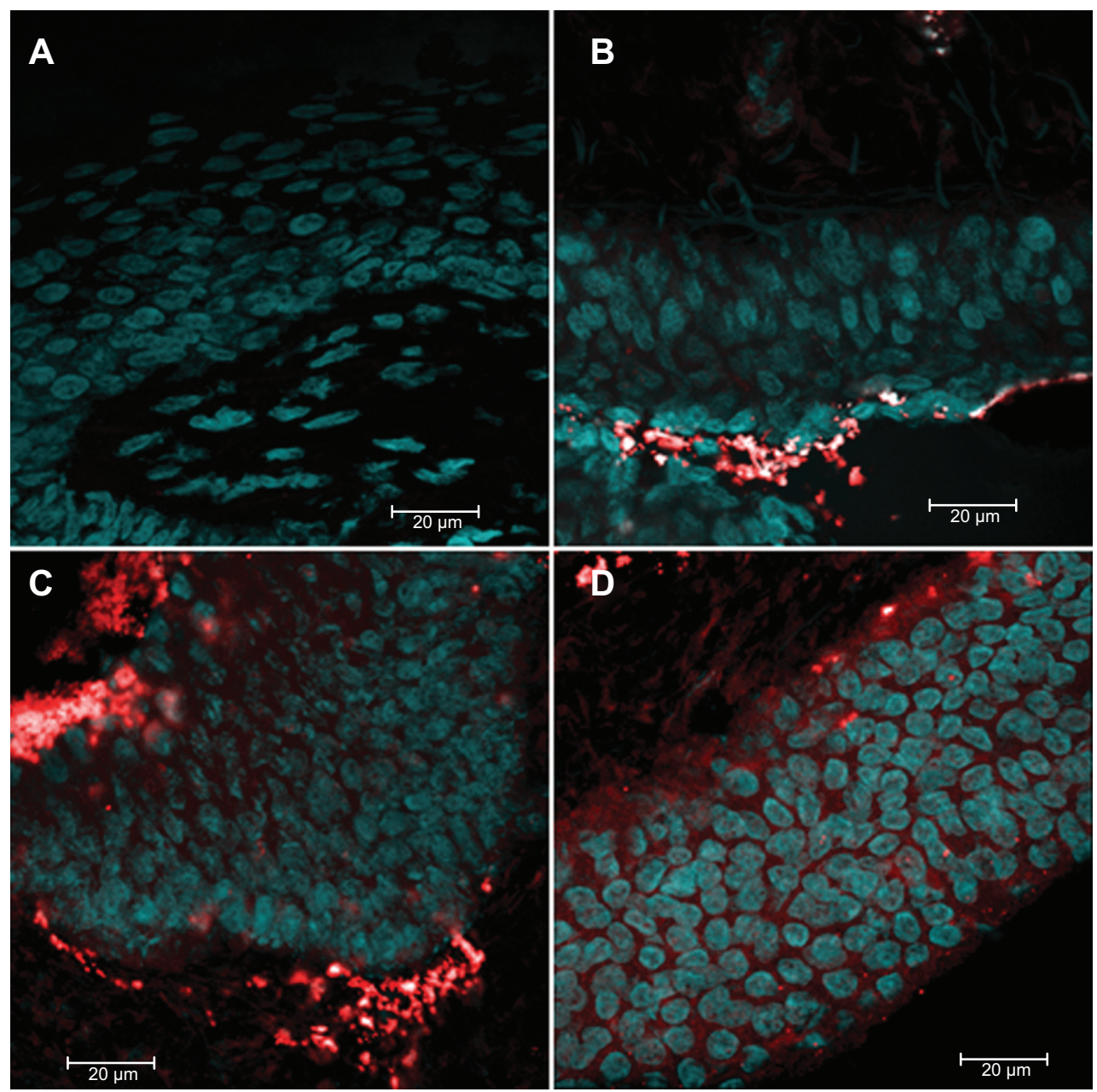

Figure 5 Confocal laser scanning microscopy images of the vaginal mucosa after application of chitosan gels containing Nile red.

Notes: $2.5 \%$ chitosan. The pictures are representatives of the measurement of three different batches: (A) blank; (B) $\mathrm{CH}-\mathrm{NR}$; (C) $\mathrm{CH}-\mathrm{RS}-\mathrm{NR}$, (D) $\mathrm{CH}-\mathrm{S}-\mathrm{NR}$. Eudragit ${ }^{\circledR}$ RS 100 and Eudragit ${ }^{\circledR} \mathrm{S} 100$ (Evonik Industries AG, Essen, Germany).

Abbreviations: $\mathrm{CH}$, chitosan hydrogel; $\mathrm{CH}-\mathrm{RS}$, chitosan hydrogel with Eudragit RS 100 nanocapsules; $\mathrm{CH}-\mathrm{S}$, chitosan hydrogel with Eudragit $\mathrm{S}$ I00 nanocapsules; NR, Nile red. 
of $\mathrm{CH}-\mathrm{NR}, \mathrm{CH}-\mathrm{RS}-\mathrm{NR}$, and $\mathrm{CH}-\mathrm{S}-\mathrm{NR}$, respectively. Figure $5 \mathrm{~B}-\mathrm{D}$ enables visual detection of fluorescence in the mucosa slices. Figure 5B shows that the application of the $\mathrm{CH}-\mathrm{NR}$ formulation led to a lower amount of fluorescence in the mucosa (small red area of the picture) compared with Figure 5C and D representing gels containing nanoencapsulated NR. Higher fluorescence was visualized in the CH-RS-NR formulation (Figure 5C) compared with $\mathrm{CH}-\mathrm{S}-\mathrm{NR}$ formulation (Figure 5D). Results based on such images are all in accordance with the penetration profile obtained with extracted samples (Figure 4).

The comparison between the chitosan gels containing cationic and anionic nanocapsules agrees with the tensile stress measurement results for mucoadhesion, where the formulations containing nanocapsules remained in contact with the vaginal mucosa for a longer time. Thus, increased contact time of these formulations containing nanocapsules leads to increased penetration of NR. Such results show that nanoencapsulated drugs can easily penetrate into the tissue.

\section{Conclusion}

Polymeric nanocapsules with cationic and anionic surface charges encapsulating NR as a lipophilic model substrate demonstrated appropriate properties for vaginal application. Chitosan gels were produced as vehicles for those nanocapsules, with a chitosan concentration of $2.5 \% \mathrm{w} / \mathrm{w}$ determined to be the most suitable for vaginal delivery. The chitosan hydrogels exhibited higher adhesion to vaginal mucosa, which is most likely related to the higher contribution of the viscous property over the elastic property of the gels. Regarding the penetration of NR into the mucosa, the results indicated that a greater amount of NR penetrated into the mucosa when it was nanoencapsulated, especially when loaded in positively charged nanocapsules, most likely due to the electrostatic interaction with the negatively charged tissue.

Considering these results, this work shows the applicability of this innovative formulation composed of $2.5 \% \mathrm{w} / \mathrm{w}$ chitosan gel, as the vehicle, and polymeric nanocapsules to vaginal drug administration. The chitosan gel and polymeric nanocapsules increase the mucoadhesion and the amount of lipophilic drug that penetrates into the tissue and might lead to a greater effect compared with the free drug. The innovative formulation can be considered as an alternative for treatments where the drug should be retained, for a long period of time, in contact with the mucosa, and also for treatments where the adverse effects of the drug, such as mucosa irritation, should be decreased, which could be achieved by the nanoencapsulation.

\section{Acknowledgment}

The authors thank Capes, CNPq, and FAPERGS for the financial support received.

\section{Disclosure}

The authors report no conflicts of interest in this work.

\section{References}

1. Sandri S, Rossi S, Ferrari F, Bonferoni MC, Muzzarelli C, Caramella C. Assessment of chitosan derivatives as buccal and vaginal penetration enhancers. Eur J Pharm Sci. 2004;21:351-359.

2. Valenta $\mathrm{C}$. The use of mucoadhesive polymers in vaginal delivery. $A d v$ Drug Deliv Rev. 2005;57:1692-1712.

3. Perioli L, Ambrogi V, Venezia L, Pagano C, Ricci M, Rossi C. Chitosan and a modified chitosan as agents to improve performances of mucoadhesive vaginal gels. Colloids Surf B Biointerfaces. 2008;66:141-145.

4. Bonferoni MC, Sandri G, Rossi S, Ferrari F, Gibin S, Caramella C. Chitosan citrate as multifunctional polymer for vaginal delivery Evaluation of penetration enhancement and peptidade inhibition properties. Eur J Pharm Sci. 2008;33:166-176.

5. Baloglu E, Senygit ZA, Karavana SY, Bernkop-Schnürch A. Strategies to prolong the intravaginal residence time of drug delivery systems. J Pharm Pharm Sci. 2009;12:312-336.

6. Bowman MC, Ballard TE, Ackerson CJ, Feldheim DL, Margolis DM, Melander C. Inhibition of HIV fusion with multivalent gold nanoparticles. J Am Chem Soc. 2008;130:6896-6897.

7. Lara HH, Ayala-Nuñez NV, Ixtepan-Turrent L, Rodriguez-Padilla C. Mode of antiviral action of silver nanoparticles against HIV-1. J Nanobiotechnology. 2010;8:1.

8. Alukda D, Sturgis T, Youan BB. Formulation of tenofovir-loaded functionalized solid lipid nanoparticles intended for HIV prevention. J Pharm Sci. 2011;100:3345-3356.

9. Patel GM, Patel PV. Novel vaginal anti-HIV drug delivery system of tenofovir disoproxil fumarate. Am J Pharm Tech Res. 2011;1:366-383.

10. Bachhav YG, Patravale VB. Microemulsion-based vaginal gel of clotrimazole: formulation, in vitro evaluation and stability studies. AAPS Pharm Sci Tech. 2009;10:476-481.

11. Schaffazick SR, Guterres SS. Caracterização e estabilidade físicoquímica de sistemas poliméricos nanoparticulados para administração de fármacos. [Physicochemical characterization and stability of the polymeric nanoparticle systems for drug administration]. Quim Nova. 2003;0:1-12. Portuguese.

12. Haas SE, Bettoni CC, de Oliveira LK, Guterres SS, Costa TD. Nanoencapsulation increases quinine antimalarial efficacy against Plasmodium Berghei in vivo. Int J Antimicrob Agents. 2009;34:156-161.

13. Contri RV, Frank LA, Kaiser M, Pohlmann AR, Guterres SS. Decreased irritation of capsaicinoids to human skin by means of nanoencapsulation. Int J Nanomedicine. 2014;9:951.

14. Hussain A, Ahsan F. The vagina as a route for systemic drug delivery. J Control Release. 2005;103:301-313.

15. Illum L, Fischer AN, Jabbal-Gill I, Davis SS. Bioadhesive starch microspheres and absorpition enhancing agents act synergistically to enhance the nasal absorption of polypeptides. Int J Pharm. 2001;222:109-119.

16. Valenta C, Auner BG. The use of polymers for dermal and transdermal delivery. Eur J Pharm Biopharm. 2204;58:279-289.

17. Contri RV, Katzer T, Ourique AF, et al. Combined effect of polymeric nanocapsules and chitosan hydrogel on the increase of capsicinoids adhesion to the skin surface. $J$ Biomed Nanotechnol. 2014;10: 820-830. 
18. Rinaudo M. Chitin and chitosan: properties and applications. Prog Polym Sci. 2006;31:603-632.

19. Sayin B, Somavarapu S, Li XW, Sesardic D, Senel S, Alpar OH. TMC-MCC (N-trimethyl chitosan-mono-N-carboxymethyl chitosan nanocomplexes for mucosal delivery of vaccines. Eur J Pharm Sci. 2009;38:362-369.

20. Wittaya-Areekul S, Kruenate J, Prahsarn C. Preparation and in vitro evaluation of mucoadhesive properties of alginate/chitosan microparticles containing prednisolone. Int J Pharm. 2006;312: 113-118.

21. Zhang S, Kawakami K. One-step preparation of chitosan solid nanoparticles by electrospray deposition. Int J Pharm. 2010;397: 211-217.

22. Berger J, Reist M, Mayer JM, Felt O, Peppas NA, Gurny R. Structure and interactions in covalently and ionically crosslinked chitosan hydrogels for biomedical applications. Eur J Pharm Biopharm. 2004;57: 19-34.

23. Meng J, Sturgis TF, Youan BB. Engineering tenofovir loaded chitosan nanoparticles to maximize microbicide mucoadhesion. Eur J Pharm Sci. 2011;44:57-67.

24. Contri RV, Katzer T, Pohlmann AR, Guterres SS. Chitosan hydrogel containing capsaicinoids-loaded nanocapsules: an innovative formulation for topical delivery. Soft Matter. 2010;8:370-385.

25. Katzer T, Chaves P, Bernardi A, Pohlmann AR, Guterres SS, Beck RC Castor oil and mineral oil nanoemulsion: development and compatibility with a soft contact lens. Pharm Dev Technol. 2014;19: 232-237.
26. Santos SS, Lorenzoni A, Ferreira LM, et al. Clotrimazole-loaded Eudragit $^{\circledR}$ RS 100 nanocapsules: preparation, characterization and in vitro evaluation of antifungal activity against Candida species. Mater Sci Eng C Mater Biol Appl. 2013;33:1389-1394.

27. Fessi H, Puisilux F, Devissaguet JP, Ammoury N, Benta S. Nanocapsule formation by interfacial polymer deposition following solvent displacement. Int J Pharm. 1989;55:R1-R4.

28. Schaffazick SR, Pohlmann AR, de Cordova CA, Creczynski-Pasa DB, Guterres SS. Protective properties of melatonin-loaded nanoparticles against lipid peroxidation. Int J Pharm. 2005;289:209-213.

29. Bonferoni MC, Rossi S, Ferrari F, Caramella C. A modified Franz diffusion cell for simultaneous assessment of drug release and washability of mucoadhesive gels. Pharm Dev Technol. 1999;4:45-53.

30. Rossi S, Bonferoni MC, Ferrari F, Caramella C. Drug release and washability of mucoadhesive gels based on sodium carboxymethylcellulose and polyacrylic acid. Pharm Dev Technol. 1999;4:55-63.

31. Contri RV, Kaiser M, Poletto FS, Pohlmann AR, Guterres SS. Simultaneous control of capsaicinoids release from polymeric nanocapsules. J Nanosci Nanotechnol. 2011;11:2398-2406.

32. Contri RV, Soares RMD, da Silveira NP, Pohlmann AR, Guterres SS. Develoment of chitosan hydrogels containing nanocapsules and characterization by oscilatory rheology. Proceedings of the XII International Macromolecular Colloquium; September 7-10; 2010; Gramado.

33. Torchilin VP. Drug targeting. Eur J Pharm Sci. 2000;11 Suppl 2: S81-S91.

34. Mallipeddi R, Rohan LC. Nanoparticle-based vaginal drug delivery systems for HIV prevention. Expert Opin Drug Deliv. 2010;7(1):37-48.
International Journal of Nanomedicine

\section{Publish your work in this journal}

The International Journal of Nanomedicine is an international, peerreviewed journal focusing on the application of nanotechnology in diagnostics, therapeutics, and drug delivery systems throughout the biomedical field. This journal is indexed on PubMed Central, MedLine, CAS, SciSearch $®$, Current Contents $\AA /$ Clinical Medicine,

\section{Dovepress}

Journal Citation Reports/Science Edition, EMBase, Scopus and the Elsevier Bibliographic databases. The manuscript management system is completely online and includes a very quick and fair peer-review system, which is all easy to use. Visit http://www.dovepress.com/ testimonials.php to read real quotes from published authors. 